\title{
ASYMPTOTIC PHEROMONE BEHAVIOR IN SWARM INTELLIGENT MANETS
}

\author{
An Analytical Analysis of Routing Behavior
}

\author{
Martin Roth and Stephen Wicker \\ Wireless Intelligent Systems Laboratory* \\ School of Electrical and Computer Engineering \\ Cornell University \\ Ithaca, New York 14850 \\ USA \\ roth@ece.cornell.edu \\ wicker@ece.cornell.edu
}

\begin{abstract}
An analytical justification is proposed for the design and global routing performance of three pheromone update methods proposed for use in Termite, a swarm intelligent routing algorithm for mobile wireless ad-hoc networks. A simple model is used in order to determine the average amount of pheromone present on a link, as well as some basic aspects of the pheromone dynamics. This includes a tendency towards a one-zero pheromone distribution favoring the better link. The pheromone update methods are investigated with the perspective that link pheromone is more an estimate of link utility than simply a routing heuristic. This allows the routing solution to be rephrased from a biological analogy to a more traditional best-metric routing terminology. A signal estimation perspective is suggested.
\end{abstract}

\section{Introduction}

Recent applications of biologically inspired algorithms to routing in mobile wireless ad-hoc networks (MANETs) have shown increased performance over traditional approaches in many critical metrics [1] [2] [3]. It remains unclear as to exactly why they work as well as they do, and how to best take advantage of their positive and negative feedback mechanisms.

This paper presents a simple analytical model of Termite, a swarm intelligent MANET routing algorithm [1]. The purpose of this model is to discover 
how individual parameters are related to each other and how they affect global metrics, such as the reliability of message delivery and adaptability to changes in the network environment. The critical element under study is pheromone. Because Termite is based on a model of social insect behavior, much of the biological terminology remains. Pheromone is a measure of the metric that the network is optimizing for; it is a measure of link or route utility. The model will first be used to characterize the behavior of pheromone on a single communications link. This will establish an intuition for determining the dynamics of pheromone in a system of two links.

\subsection{Previous Work}

Routing algorithms are often difficult to formalize into mathematics; they are instead tested using extensive simulation [14]. The interaction between parameters is unclear, as are their global effects, or even how the values should be determined for optimal performance.

Swarm intelligent routing algorithms lend themselves to mathematical analysis. Their routing update and decision procedures are mathematical functions themselves. One of the earliest works on swarm intelligent routing is by Schoonderwoerd et al. on the Ant Based Control (ABC) algorithm [9]. This algorithm is for a wired circuit switched network, such as a telephony network. $\mathrm{ABC}$ was later modeled analytically in [6]. This work demonstrates and proves the behavior of pheromone and its effect on global system performance in $\mathrm{ABC}$.

Substantially more work has been done to characterize the behavior of such systems by means of simulation. Many were inspired by the Ant Colony Optimization (ACO) algorithm [10]. A number of routing algorithms for packet switched networks are also available based on this framework [11]. These ideas have also been adapted to the field of mobile wireless ad-hoc networks [1] [3] [5]. Finally, a great deal of work has been done with the original biologically inspired models. This work spans several fields, including experimental biology, theoretical biology, and the various disciplines of engineering which apply the models derived by the former. Some summaries may be found in [12] and [13].

\subsection{Structure of Paper}

Section II gives a brief introduction to the MANET routing problem, as well as a review of the Termite routing algorithm. An analytical model of a MANET is presented in Section III. This model captures some of the most critical aspects of the network, and allows for easy integration with the routing equations used by Termite. Section IV illustrates in analytical detail the average behavior of pheromone on a link, both alone and coupled with other links. Comparisons 
are made between three pheromone update methods. Section V analyzes the results of previous section and explains earlier experimental results. Section VI concludes the paper with a note about future work and final remarks.

\section{Termite Routing for MANETs}

This section will give a short introduction to the routing problem in mobile wireless ad-hoc networks. A brief review of the Termite swarm intelligent routing algorithm follows. The section will conclude with some problems exhibited by the algorithm, which will motivate the goals of the analysis.

\subsection{A Short Introduction to Ad-Hoc Networks}

A mobile wireless ad-hoc network is a collection of mobile computers able to communicate wirelessly with others who are within radio range. Each computer, or node, is able to forward messages for others such that any pair of nodes in the network are able to communicate with each other. Using this forwarding technique, a member of the network with only minimal communications capability is able to take advantage of the resources offered by the sum. No installed infrastructure is necessary for the network to operate.

Due to node mobility, the topology of the network changes often. Some of the outstanding problems include network scalability, the speed with which a routing algorithm is able to adapt to a new topology, the amount of control traffic needed to maintain network connectivity, and data packet delivery reliability [14].

\section{$2.2 \quad$ Termite}

Termite is a distributed routing algorithm for mobile wireless ad-hoc networks [1]. It is designed using the swarm intelligence framework in order to achieve better adaptivity, lower control overhead, and lower per-node computation. The algorithm is inspired by the hill building behavior of termites.

The Termite algorithm is explained in detail in [1] and updated in [2], however it may be described simply as follows. Each node in the network has a specific pheromone scent. As packets move through the network on links between nodes, they are biased to move in the direction of destination pheromone gradients. Packets follow this gradient while laying pheromone for their source on the links that they traverse. The amount of pheromone deposited by a packet on a link is equal to the utility of its traversed path. Using this method of pheromone updating, consistent pheromone trails are built through the network. Changes in the network environment, such as topological or path quality changes, are accounted for by allowing pheromone to decay over time. This requires paths to be continuously reinforced by new traffic; new information about the network is added to links. Each node records the amount of 
pheromone that exists for each destination on each of its links. This creates a routing table similar to those found in traditional link-state routing algorithms.

Pheromone Update. The pheromone update equation is a function which updates the pheromone of the packet source at a node upon its arrival. The update function shown here is the traditional approach and is known as the $\gamma$ Pheromone Filter. Pheromone on all links decays simultaneously upon packet arrival, and proportionally to packet interarrival times. This is known as continuous pheromone decay and was originally presented in [3]. This idea is extended in this work by also decaying pheromone when it is checked to send a packet. In general, pheromone is decayed whenever it is observed. This allows the system to truly keep track of the real time deterioration of the reliability of available information about the network. Each packet maintains the total utility of the path it has traversed (updated at each node visited), $\gamma$, and deposits this amount of pheromone on each link. The $\gamma$ Pheromone Filter pheromone update equation is,

$$
\begin{aligned}
\forall i P_{i, s}^{n} & =P_{i, s}^{n} \cdot e^{-\left(t-t_{s, o b s}^{n}\right) \tau} \\
P_{r, s}^{n} & =P_{r, s}^{n}+\gamma
\end{aligned}
$$

where $P_{i, s}^{n}$ is the amount of pheromone from source node $s$, on the link from neighbor node $i$, at node $n$. The previous hop of the packet is node $r$. The variable $\gamma$ is the amount of pheromone carried by the packet, which will vary from packet to packet depending on its path. The time $t_{s, o b s}^{n}$ is the last instant that the pheromone from source node $s$ at node $n$ was observed, either due to packet sending or packet receiving. The pheromone decay rate is $\tau$.

Forwarding Equation. The forwarding equation is used to determine the probability of using a link based on the amount of pheromone on it.

$$
p_{i, d}^{n}=\frac{\left(P_{i, d}^{n}+K\right)^{F}}{\sum_{j=1}^{N_{n}}\left(P_{j, d}^{n}+K\right)^{F}}
$$

where $p_{i, d}$ is the probability of using neighbor node $i$ in order to get to destination node $d$, at node $n . N_{n}$ is the number of neighbors of node $n . K \geq 0$ is the pheromone threshold and $F \geq 0$ is the pheromone sensitivity.

Termite Summary. Termite has been shown to perform well, especially in regions of high node mobility [2] [1]. However, packets often take substantially longer than necessary paths. In part, this is required in order to maintain current pheromone through the network, but this behavior can also generate significant resource inefficiencies. 


\section{The Model}

A model of an ad-hoc network is presented which will be used to evaluate the behavior of the pheromone update methods. The network is modeled as two communicating nodes with two independent paths available between them. These paths abstract all other connections between the two nodes, including additional nodes, mobility issues, or communications effects. The physical structure is shown in Figure 1, and is the same as that used in [6].

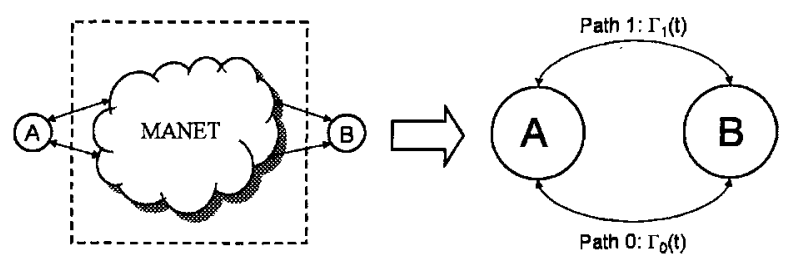

Figure 1. Diagram of the MANET Model

Each node sends packets to the other with iid exponentially distributed interarrival times. The average rate at which node $A$ sends to $B$ is $\lambda_{A}$, and $\lambda_{B}$ in the opposite direction. Each node is also able to decay the pheromone on its links independently. The decay rates at each node are $\tau_{A}$ and $\tau_{B}$.

Each path $i$ has a utility characterized by a non-negative random process $\Gamma_{i}(t)$ with mean $\mu_{i}(t)$. The pheromone contained in a packet arriving on a link, $\gamma$, is a sample of that process. $\Gamma$ is non-stationary since link utilities change over time due to mobility and other effects. $\Gamma$ is considered to be stationary in this work for ease of analysis. Since each packet passes through the network indepedently of all other packets, there is no correlation between successive samples of the link utility process. The forwarding equation independently considers each packet.

\section{Pheromone Update Analysis}

This section analyzes the amount of pheromone found on a link. The results will explain the performance of the $\gamma$ Pheromone Filter $(\gamma$ PherFilt), Joint Decay IIR Filter (IIR2), and pDijkstra pheromone update methods, which are reprinted in Figure 2 from [2]. Namely, why $\gamma$ PherFilt achieves such high goodput while being slow to adapt at high mobility, why pDijkstra adapts better, and why IIR2 is able to achieve the highest performance with regards to the Delivery Efficiency metric.

Goodput is the fraction of successfully delivered data packets, while the latter metric also includes the ratio of the achieved path metric to the best possible available at the time. 

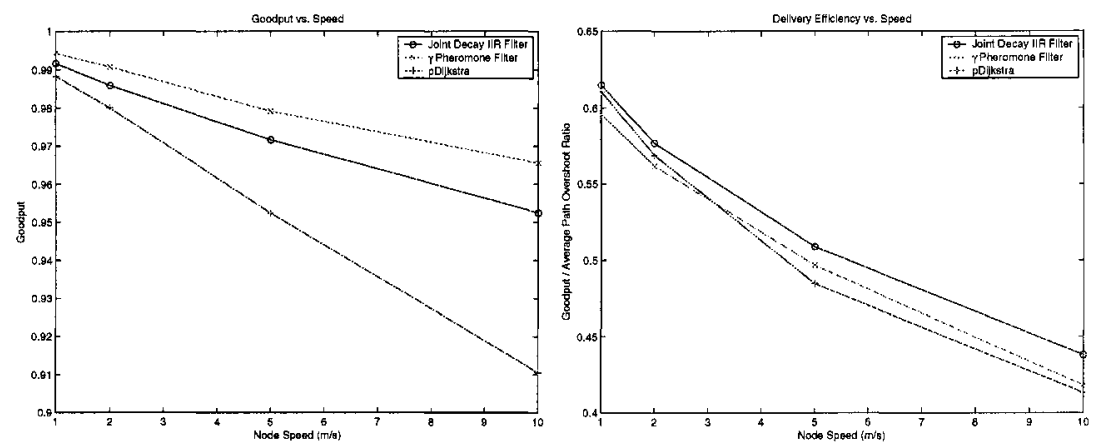

Figure 2. Performance vs. Pheromone Update Method

\subsection{Single Link Pheromone}

A system of one link is first considered in order to give insight on the more interesting system of two links. A formula is established for the time average pheromone deposited on a link, given that the packet arrival rate is poisson distributed with mean $\lambda$ [packets/second]. The decay rate, $\tau$ [1/second] remains constant. The poisson packet arrival rate assumption implies that the packet interarrival times are exponentially distributed with mean, $\lambda^{-1}$ [seconds/packet]. The average value of the received pheromone is $E \Gamma=\mu$. The amount of pheromone on the link before packets begin arriving is $P_{0}$.

By applying the pheromone update equation $n$ consecutive times, an expression is derived for the amount on a link given that $n$ packets have arrived, $P(n)$. The packet interarrival time of the $n$th packet is independently and identically distributed, $t_{n}$.

$$
\begin{aligned}
P(n) & =\left(\left(\left(P_{0} e^{-t_{1} \tau}+\mu\right) \cdot e^{-t_{2} \tau}+\mu\right) \cdot \ldots\right) \cdot e^{-t_{n} \tau}+\mu \\
& =P_{0} e^{-\left(\sum_{i=1}^{n} t_{i}\right) \tau}+\mu\left[\sum_{i=2}^{n} e^{-\left(\sum_{j=i}^{n} t_{j}\right) \tau}\right]
\end{aligned}
$$

The expectation of $P(n)$ with respect to packet interarrival time is found according to standard methods.

$$
E P(n)=\frac{P_{0} \lambda^{n}}{(\lambda+\tau)^{n}}+\sum_{i=0}^{n-1} \frac{\mu \lambda^{i}}{(\lambda+\tau)^{i}}
$$

In order to simplify Equation 4, substitute $\beta=\frac{\lambda}{\lambda+\tau}$, and further reduce the expression.

$$
E P(n)=P_{0} \beta^{n}+\mu\left(\frac{1-\beta^{n}}{1-\beta}\right)
$$


To arrive at an expression for the expected amount of pheromone on a link over time, note that the number of packet arrivals, $n$, within a given time, $t$, is distributed according to the poisson distribution with parameter, $\lambda t$.

$$
\begin{aligned}
E P(t) & =\sum_{n=0}^{\infty}[\text { poisson }(\lambda t, n)][E P(n)] \\
& =\sum_{n=0}^{\infty}\left[e^{-\lambda t} \frac{(\lambda t)^{n}}{n !}\right]\left[P_{0} \beta^{n}+\mu\left(\frac{1-\beta^{n}}{1-\beta}\right)\right] \\
& =\frac{\mu}{1-\beta}+e^{-\frac{\lambda \tau t}{\lambda+\tau}}\left(P_{0}-\frac{\mu}{1-\beta}\right)
\end{aligned}
$$

The long term behavior of the link pheromone is defined as its mean.

$$
\begin{aligned}
\lim _{t \rightarrow \infty} E P(t) & =\frac{\mu}{1-\beta} \\
& =\frac{\mu(\lambda+\tau)}{\tau} \\
& \stackrel{\text { def }}{=} E P
\end{aligned}
$$

A similar analysis shows the variance of the link pheromone,

$$
V A R(P)=\frac{\mu^{2} \lambda}{2 \tau}
$$

Scale Invariance. The ratio of $\lambda$ and $\tau$ is a scale invariant parameter in this system. Primarily characterized by the expected decay factor in Equation 4, the expected pheromone on a link may be held constant as long as $\frac{\lambda}{\tau}$ remains the same. The scale invariant parameter is proportional to $\beta$.

\subsection{Two Link Pheromone}

The following analysis shows the average value of pheromone on each link in a two link system. The two link system is that described by the model in Section III. The $\gamma$ Pheromone Filter, Joint Decay IIR Filter, and pDijkstra update methods are reviewed. Each generates different pheromone dynamics and maintains varying amounts of link pheromone in equilibrium. Each method falls into a one-zero pheromone distribution in the correct parameter space, which echoes previous results from an analysis of the Ant Based Control routing algorithm [6].

A system of equations is presented which recursively compute the mean pheromone at each node on each link, $P_{0, B}^{A}, P_{1, B}^{A}, P_{0, A}^{B}$, and $P_{1, A}^{B}$. Pheromone changes either when it is checked in order to send a packet, or when it is updated due to packet arrival. The total rate at which pheromone is observed is 
$\lambda=\lambda_{A}+\lambda_{B}$. The Pheromone Check proceedure only decays the pheromone and accounts for the fraction of the instances when a packet must be sent. During Packet Arrival, which accounts for the fraction of instances that a packet arrives, the pheromone is not only decayed, but also incremented if the packet arrives on the correct link.

The average amount by which pheromone decays between observations has already been implicitly derived in Equation 4 . Suppose random variable $Y$ is the interarrival time between packets and is distributed exponentially with mean $\lambda^{-1}$, as described in the model definition. Random variable $X$ is defined such that $X=e^{-Y \tau}$, which describes the fraction of pheromone decayed in between packet arrivals. Its probability distribution function is, $f_{X}(x)=$ $\frac{\lambda}{\tau} x^{\left(\frac{\lambda}{\tau}-1\right)}$ where $0 \leq x \leq 1 . E X=\frac{\lambda}{\lambda+\tau}=\beta$.

The $\gamma$ Pheromone Filter. The $\gamma$ Pheromone Filter has been defined previously. The example below develops the average link pheromone equation for $P_{0, B}^{A}$ based on the previous description of pheromone influences.

$$
\begin{aligned}
P_{0, B}^{A} & =\text { Pheromone Check } \\
& + \text { Update Not On This Link +Update On This Link } \\
P_{0, B}^{A} & =\frac{\lambda_{A}}{\lambda}\left[\left(\frac{\lambda}{\lambda+\tau_{A}}\right) P_{0, B}^{A}\right] \\
& +\frac{\lambda_{B}}{\lambda}\left\{p_{1, A}^{B}\left[\left(\frac{\lambda}{\lambda+\tau_{A}}\right) P_{0, B}^{A}\right]+p_{0, A}^{B}\left[\left(\frac{\lambda}{\lambda+\tau_{A}}\right) P_{0, B}^{A}+\mu_{0}\right]\right\} \\
& =\left(\frac{\lambda}{\lambda+\tau_{A}}\right) P_{0, B}^{A}+\left(\frac{\lambda_{B}}{\lambda}\right) p_{0, A}^{B} \mu_{0}
\end{aligned}
$$

where $p_{0, A}^{B}$ is the link probability described in Equation 2. The evolution of pheromone on a link at one node as packets are received is illustrated in Figure 3.

Note that when $K=0$, the asymptotic probability mass function between the two links follows a one-zero distribution; the algorithm uses the better link exclusively. The reason for this is that when a packet arrives at a node, its link is positively reinforced, while all other links at that node are negatively reinforced; pheromone decays on all links but is only replaced on one. Because packets are also biased towards pheromone, this produces a strong positive feedback which eventually transfers all traffic to the link with the highest utility. With no pheromone threshold there is no incentive to use a lesser link. Consequently the probability of using it disappears. Under these conditions, the dominant link follows the behavior of the single link. Only when $\mu_{0}=\mu_{1}$ do the links have equal probability.

With $K>0$, traffic is allowed to be forwarded over all links, regardless of their utility. Currently bad links may be tested on occasion for a change 

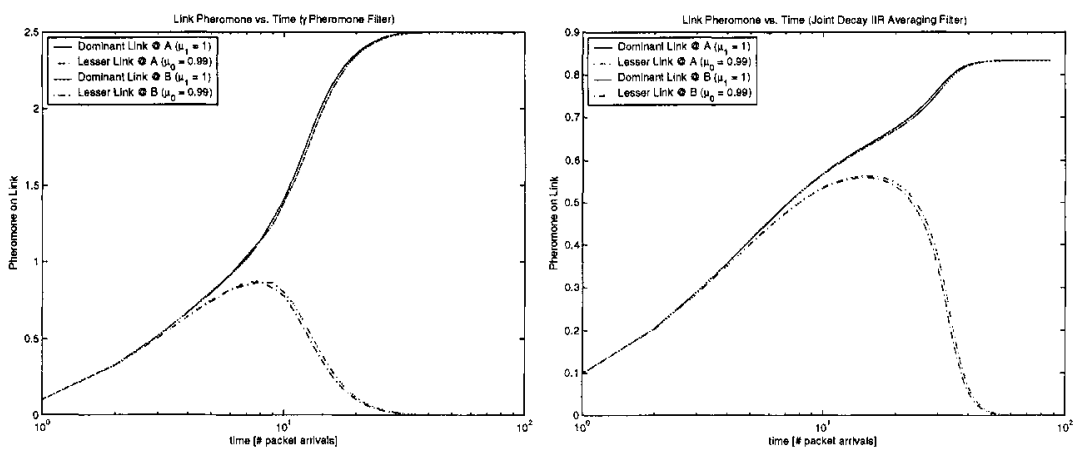

Figure 3. Pheromone vs. Packets Received $\left(\beta=\frac{\grave{\lambda}=2}{2+\tau=1}=\frac{2}{3}, K=0, F=5, R=0.5\right.$ )

in utility. This allows for a measure of adaptivity, however $K$ must be set appropriately in order to allow for links to be tested often enough. Too few tests of other links will not overcome the positive feedback towards to the dominant link.

The mean link pheromone on the dominant link in the case of a one-zero pheromone distribution can be found by setting the probability of using the dominant link to one and solving for the remaining pheromone. Solving this case for $\tilde{P}_{0, B}^{A}$,

$$
\tilde{P}_{0, B}^{A}=\frac{\lambda_{B}\left(\lambda+\tau_{A}\right)}{\lambda \tau_{A}} \mu_{0}
$$

Joint Decay IIR Filter (IIR2). The Joint Decay IIR Filter implements a simple one-tap infinite impulse response (IIR) averaging filter with joint pheromone decay. The inspiration for such an approach comes from the Single Link Pheromone analysis. There, the expected link pheromone may be represented by the sum shown by Equation 11. This is a reexpression of Equation 4. Indices represent packet arrivals; $\gamma(n)$ is the amount of pheromone contained in the $n$th packet, and $P(n)$ is the expected pheromone after its arrival. Assume $P_{0}=0$ and $B(n)=\beta^{n}=\left(\frac{\lambda}{\lambda+\tau}\right)^{n}, n \geq 0$.

$$
\begin{aligned}
P(n) & =P_{0} \beta^{n}+\sum_{i=0}^{n-1} \gamma(n-i) \beta^{i} \\
& =\gamma(n) * B(n)
\end{aligned}
$$

In this interpretation, the series $B(n)$ is the average impulse response of the $\gamma$ Pheromone Filter in the single link case. This is a simple unnormalized one tap IIR averaging filter. IIR 2 is derived from $\gamma$ PherFilt by normalizing the received pheromone to create a version of the well known one-tap IIR averaging filter. 
Thus, the IIR2 pheromone update equation is,

$$
\begin{aligned}
\forall i P_{i, s}^{n} & =P_{i, s}^{n} \cdot e^{-\left(t-t_{s, o b s}^{n}\right) \tau} \\
P_{r, s}^{n} & =P_{r, s}^{n}+\left[1-e^{-\left(t-t_{i, s}^{n}\right) \tau}\right] \gamma
\end{aligned}
$$

where $t_{i, s}^{n}$ is the last time a packet arrived from source $s$ from neighbor $i$ at node $n$.

The analysis of IIR 2 may be treated similarly to the analysis of $\gamma$ PherFilt. IIR2 requires that arriving pheromone be normalized according to the time since a packet last arrived on that link. The result for $P_{0, B}^{A}$ is shown.

$$
P_{0, B}^{A}=\left(\frac{\lambda}{\lambda+\tau_{A}}\right) P_{0, B}^{A}+\left(\frac{\lambda_{B}}{\lambda}\right)\left[1-\left(\frac{p_{0, A}^{B} \lambda_{B}}{p_{0, A}^{B} \lambda_{B}+\tau_{A}}\right)\right] p_{0, A}^{B} \mu_{0}
$$

Time pheromone evolutions is also shown in Figure 3. Mean link pheromone in the one-zero distribution case is shown,

$$
\tilde{P}_{0, B}^{A}=\frac{\lambda_{B}\left(\lambda+\tau_{A}\right)}{\lambda\left(\lambda_{B}+\tau_{A}\right)} \mu_{0}
$$

pDijkstra. The simulations of [2] show that the previous pheromone update methods provide the best results with a high pheromone sensitivity, $F$. The results in Figure 3 above show that a high sensitivity will quickly force the links pheromone towards a one-zero distribution and thus to the exclusive use of the best link (save for any threshold). The determination and selection of a best neighbor link to a destination is reminiscent of typical link-state routing such as Dijkstra's algorithm [15]. pDijkstra is developed where the pheromone update equation is,

$$
\begin{array}{ll}
\forall i & P_{i, s}^{n}=P_{i, s}^{n} \cdot e^{-\left(t-t_{s, o b s}^{n}\right) \tau} \\
\text { if } & P_{r, s}^{n}<\gamma, P_{r, s}^{n}=\gamma
\end{array}
$$

Similarly to Dijkstra's algorithm, if a link with higher utility is found, then the link pheromone is updated. All pheromone decays concurrently as in the previous methods.

The pDijkstra pheromone update method cannot be analyzed in a similar way because it is nonlinear. For the purposes of the analysis presented here it suffices to note that the link pheromone is upper bounded by the utility of the link.

$$
\tilde{P}_{0, B}^{A} \leq \mu_{0}
$$

\section{Analysis}

As shown in the simulation results of Figure 2, $\gamma$ PherFilt has the best goodput performance but poor ability to track changes in the network. pDijkstra is 
able to do better in delivery efficiency, while IIR2 scores best in this regard. These results can be explained based on the analysis in the previous section.

$\gamma$ PherFilt maintains the largest equilibrium pheromone level on the best link. Since pheromone takes time to decay, this allows it to route on a particular link longer in case the underlying link metric change; large amounts of pheromone implies a large link forwarding probability. Due to this hysteresis effect on pheromone decay, suboptimal links are used longer, which prevents the algorithm from adapting quickly. In essence, $\gamma$ PherFilt tends to use a known good route, and thus achieves a high goodput, but it is unwilling to change that route in the face of varying route and link metrics.

IIR2 and pDijkstra see less goodput but higher adaptivity compared to $\gamma$ PherFilt, both for similar reasons. They maintain less pheromone on the links and because of this are able to adapt to changes faster. Less pheromone requires less time to decay. Note that $\tilde{P}_{I I R 2}<\tilde{P}_{\gamma P h e r F i l t}$ due to an additional term of $\lambda \lambda_{B}$ in the demoninator of the former. Differences in pheromone between links is relatively less than with $\gamma$ PherFilt, thus the link probabilities are also less decisive during transition periods while the algorithm is choosing a new link. This leads to wandering packets which eventually timeout in the network and result in lower goodput.

It is also important to note the overall superior performance of IIR2 was derived from a simple application of linear filtering. The pheromone delivered on a packet is considered a measure of the packet's path, and it is averaged to create an expectation of the utility of that path to the destination.

\section{Conclusion}

This paper has presented an extension of an analytical model of a mobile wireless ad-hoc network. This model was used to investigate the properties of the Termite swarm intelligent MANET routing algorithm. The mean pheromone on a single link and in a system of two links were determined. These results were compared for three different pheromone update methods. Relationships between parameters were explored and scale invariance was found. It is shown that the amount of pheromone put on a link influences performance, not only in goodput, but also in adaptability. The analysis also revealed a linear filtering perspective in which link utility is directly estimated with the pheromone rather than simply using it as a routing heuristic.

Future work in this area should more completely characterize the dynamics of the pheromone update methods shown here. While there exists a good intuition for their behavior, a true formalization is necessary. An understanding of exactly what parameters allow a zero-one distribution, how long it takes to arrive at said distribution, and what conditions allow the system to evolve out of the distribution if link costs change would be helpful. 


\section{References}

[1] M. Roth, S. Wicker, Termite: Emergent Ad-Hoc Networking, The Second Mediterranean Workshop on Ad-Hoc Networks, 2003.

[2] M. Roth, S. Wicker, Performance Evaluation of Pheromone Update Methods in Termite, in preparation.

[3] M. Gunes, M. Kahmer, I. Bouazizi, Ant Routing Algorithm (ARA) for Mobile Multi-Hop Ad-Hoc Networks - New Features and Results, The Second Mediterranean Workshop on Ad-Hoc Networks, 2003.

[4] M. Gunes, U. Sorges, I. Bouazizi, ARA - The Ant-Colony Based Routing Algorithm for MANETs, Proceedings of the ICPP Workshop on Ad Hoc Networks (IWAHN 2002), IEEE Computer Society Press, 2002, 79-85.

[5] M. Heissenbuttel, T. Braun, Ants-Based Routing in Large Scale Mobile AdHoc Networks, Kommunikation in Verteilten Systemen (KiVS), 2003.

[6] D. Subramanian, P. Druschel, J. Chen, Ants and Reinforcement Learning: A Case Study in Routing in Dynamic Networks, Proceedings of the International Joint Conference on Artificial Intelligence, 1997.

[7] G. Di Caro, M. Dorigo, Mobile Agents for Adaptive Routing,Technical Report, IRIDIA/97-12, Universit Libre de Bruxelles, Belgium, 1997.

[8] B. Baran, R. Sosa, A New Approach for AntNet Routing, Proceedings of the Ninth International Conference on Computer Communications and Networks, 2000.

[9] R. Schoonderwoerd, O. Holland, J. Bruten, L. Rothkrantz, Ant-Based Load Balancing In Telecommunications Networks, Adaptive Behavior, 1996.

[10] M. Dorigo, G. Di Caro, L. M. Gambardella, Ant Algorithms for Discrete Optimization, Artificial Life, Vol. 5, No. 2, 1999.

[11] K. M. Sim, W. H. Sun, Ant Colony Optimization for Routing and LoadBalancing: Survey and New Directions, IEEE Transactions on Systems, Man, and Cybernetics - Part A: Systems and Humans, Vol. 33, No. 5, September 2003.

[12] E. Bonabeau, M. Dorigo, G. Theraulaz, Swarm Intelligence: From Natural to Artificial Systems, Oxford University Press, 1999.

[13] M. Resnick, Turtles, Termites, and Traffic Jams: Explorations in Massively Parallel Microworlds, Bradford Books, 1997.

[14] J. Broch, D. A. Maltz, D. B. Johnson, Y. Hu, J. Jetcheva, A Performance Comparison of Multi-Hop Wireless Ad Hoc Network Routing Protocols, Proceedings of the Fourth Annual ACM/IEEE International Conference on Mobile Computing and Networking, 1998.

[15] E. Dijkstra, A note on two problems in connection with graphs, Numerische Mathematik, Vol. 1, 269-271, 1959. 